Section XI

Treatment of Helicobacter pylori

Infection 
Klaus Langeheinecke (Hrsg.)

Peter Jany

Eugen Sapper

\section{Thermodynamik für Ingenieure}

\section{Ein Lehr- und Arbeitsbuch}

für das Studium

5., verbesserte Auflage

Mit 242 Abbildungen und 46 Tabellen 
Bibliografische Information Der Deutschen Bibliothek

Die Deutsche Bibliothek verzeichnet diese Publikation in der Deutschen Nationalbibliografie; detaillierte bibliografische Daten sind im Internet über <http://dnb.ddb.de> abrufbar.

\section{Der Herausgeber}

Prof. Dr.-Ing. Klaus Langeheinecke, Fachhochschule Ravensburg-Weingarten, Weingarten/Württ.

\section{Die Autoren}

Prof. Dr.-Ing. Klaus Langeheinecke, Fachhochschule Ravensburg-Weingarten, Weingarten/Württ. Prof. Dr.-Ing. Peter Jany, Fachhochschule Ravensburg-Weingarten, Weingarten/Württ.

Prof. Dr.-Ing. Eugen Sappert, Fachhochschule Konstanz, Konstanz

\section{Auflage 1993}

2., vollständig überarbeitete Auflage 1999

3., durchgesehene Auflage Mai 2001

4., überarbeitete und erweiterte Auflage Juni 2003

5., verbesserte Auflage Oktober 2004

Alle Rechte vorbehalten

(C) Springer Fachmedien Wiesbaden 2004

Ursprünglich erschienen bei Friedr. Vieweg \& Sohn Verlag/GWV Fachverlage GmbH, Wiesbaden, 2004

www.vieweg.de

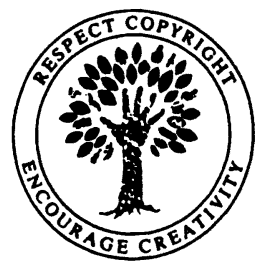

Das Werk einschließlich aller seiner Teile ist urheberrechtlich geschützt. Jede Verwertung außerhalb der engen Grenzen des Urheberrechtsgesetzes ist ohne Zustimmung des Verlags unzulässig und strafbar. Das gilt insbesondere für Vervielfältigungen, Übersetzungen, Mikroverfilmungen und die Einspeicherung und Verarbeitung in elektronischen Systemen.

Umschlaggestaltung: Ulrike Weigel, www.CorporateDesignGroup.de

Technische Redaktion: Hartmut Kühn von Burgsdorff, Wiesbaden

Gedruckt auf säurefreiem und chlorfrei gebleichtem Papier.

Additional material to this book can be downloaded from http://extra.springer.com. 


\section{Vorwort}

Die Technische Thermodynamik gehört zu den Grundlagen des Maschinenbaues, der Verfahrenstechnik und verwandter Ingenieurwissenschaften. Für Studierende an Fachhochschulen, Technischen Hochschulen und Universitäten, ferner an Höheren Technischen Lehranstalten, Technikerschulen und Berufsakademien im gesamten deutschsprachigen Raum ist dieses Buch geschrieben, zur Nutzung in und neben den Lehrveranstaltungen. Der Umfang orientiert sich an dem, was an Grundlagen für weiterführende Lehrveranstaltungen erforderlich ist. Zum Selbststudium in der Weiterbildung und beim Wiedereinstieg empfiehlt sich das Lehrbuch durch seinen Aufbau auch für bereits Berufstätige.

Ausführliche Texte, zahlreiche bildliche Darstellungen, durchgerechnete Beispiele, viele Fragen und Übungsaufgaben mit Lösungen zur aktiven Beschäftigung verdeutlichen die Denkweisen, Methoden und Werkzeuge der Thermodynamik. Vor allem wird die Fachsprache vermittelt, die sich oft als Hindernis auf dem Weg zum Verstehen der Thermodynamik erwiesen hat, aber dafür und zum Lesen von Fachliteratur unerläßlich ist. In umfangreichen Tabellen sind notwendige Daten zusammengestellt, ergänzt durch ein MOLLIER- $h, s$-Diagramm für Wasserdampf und ein MOLLIER- $p, h$-Diagramm für R134a.

Ein ausführliches Sachwortverzeichnis leitet schnell zu den gesuchten Textstellen und gibt die Sachworte auch in englischer Sprache wieder. Auf eıner CD-ROM findet der Leser das entsprechende englischdeutsche Sachwortverzeichnis, die Ergebnısse zu den Fragen und Übungen, Rechenprogramme zu Wärmeübertragung, Gas-Dampf-Gemischen und Verbrennung sowie für Stoffwerte, ferner eine als Formelsammlung und zur Wiederholung zu verwendende Kurzfassung des Lehrtextes THERMODYNAMIK MEMORY und ein umfangreiches alphabetisches, attraktiv nutzbares THERMODYNAMIK GLOSSAR mit über 700 Stichwörtern.

Die Lehre der Thermodynamik war bislang weitgehend durch die Felder „Geschlossenes System“, „Ideales Gas“ und „Reversible Prozesse“ geprägt. Technische Prozesse laufen jedoch im allgemeinen in offenen Systemen ab, über deren Grenzen Stoff- und Energieströme übertragen werden und in denen häufig Phasenwechsel und nicht vemachlässigbare Dissipationsphänomene auftreten. Daher wird das offene System so früh wie möglich vorgestellt, mit Stromgrößen und Bilanzansätzen gearbeitet. Die verschiedenen Energie- und Leistungsarten werden begrifflich klar unterschieden. Dabei wird auf die Wärme, ihre unterschiedlichen Transportmechanismen und ihre Freisetzung durch Verbrennung besonders eingegangen. Wegen oft unzureichender Vorkenntnisse in der Physik werden Phasenwechsel und das gesamte Zustandsfeld bereits am Anfang dargestellt. Die dazu notwendigen Gedankenexperimente bauen auf Alltagsbeobachtungen auf. Dabei wird in den Umgang mit Zustandsdiagrammen und Dampftafeln eingeführt und dem Modell „Ideale Gase“ der richtige Platz angewiesen.

Das Buch geht im Kern zurück auf das in langer Lehrtätigkeit entwickelte Vorlesungsmanuskript des Herausgebers, das im Rahmen des CAT-Projektes seine erste gedruckte Form fand. Die Professoren W. Schnabel, Dr. G. Kurz und Dr. G. Kürz sowie Ing. (grad.) P. Stotz haben damals teils schreibend, teils erprobend und beratend mitgewirkt. Für das Buchmanuskript konnten zusätzlich Prof. Dr. Eugen Sapper (Konstanz), der jedoch noch während der Bearbeitung verstarb, und Prof. Dr. Peter Jany (Weingarten/Württ.) sowie Dipl.-Ing. Heinz Millner (Dornbirn/Vorarlberg) gewonnen werden. Die sorgfältige Ausführung der Zeichnungen übernahm Dipl.-Ing. (FH) Wolf-Dieter Schnell (Langenargen/Bodensee).

Während das Lehrbuch für die zweite Auflage gründlich durchgesehen, überarbeitet, korrigiert und ergänzt wurde, konnten sich die Autoren bei der dritten bis fünften Auflage auf wenige Änderungen beschränken. Hinzugefügt wurde die englische Fassung des Sachwortverzeichnisses. Hinweise von Fachkollegen und Studierenden sind jeweils dankbar verwertet worden. Herausgeber, Mitautoren und Verlag danken allen Beteiligten, die zum Gelingen der fünf Auflagen des Lehrbuches beigetragen haben. Ein besonderer Dank gilt ihren Familien, die wegen des Buches so oft auf sie verzichten mussten. 


\section{Inhaltsverzeichnis}

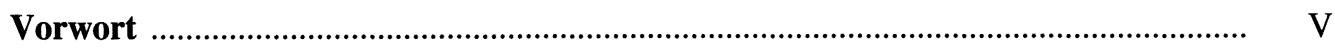

Verzeichnis der Beispiele .................................................................................... IX

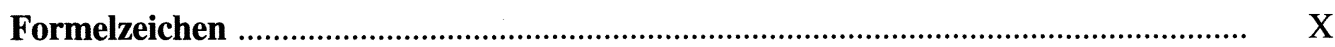

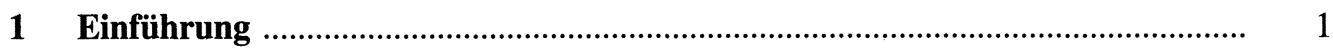

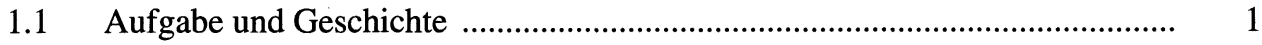

1.2 Zur Lehrveranstaltung ............................................................................ 3

1.3 Physikalische Größen und Größengleichungen .................................................. 3

$1.4 \quad$ Fragen und Übungen ......................................................................................... 7

2 Die Systeme und ihre Beschreibung ………………………………………..... 8

$2.1 \quad$ Systeme und Energien .......................................................................................

2.2 Gleichgewicht und Beharrungszustand ……….......................................... 13

2.3 Stoff und Menge ......................................................................................... 16

2.4 Zustand, Zustandsgrößen und Zustandsdiagramme ........................................ 19

$2.5 \quad$ Druck, Temperatur, Energie ........................................................................ 21

2.6 Zustandsänderungen, Prozesse ……………………………………………... 25

$2.7 \quad$ Fragen und Übungen ............................................................................... 29

3 Stoffeigenschaften ...................................................................................... 32

3.1 Thermische Dehnung ............................................................................... 32

3.2 Verdampfen und Verflüssigen .................................................................... 33

3.3 Kritischer Punkt ...................................................................................... 37

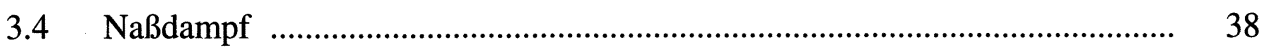

3.5 Erstarren, Sublimieren, Tripelzustände ……………………………………... 44

3.6 Dämpfe und Gase ................................................................................... 50

$3.7 \quad$ Stoffgemische ......................................................................................... 55

$3.8 \quad$ Fragen und Übungen .................................................................................. 57

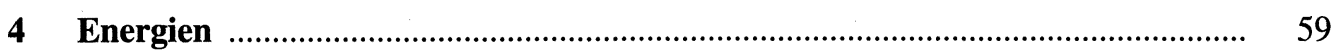

$4.1 \quad$ Energiegrößen und Erster Hauptsatz ……………......................................... 59

$4.2 \quad$ Arbeit und Arbeitsleistung ................................................................................. 64

4.3 Wärme, Wärmestrom und Innere Energie ………………………………........ 69

$4.4 \quad$ Enthalpie und Enthalpiestrom .................................................................. $\quad 70$

4.5 Energiebilanz von Kreisprozessen ............................................................... $\quad 74$

4.6 Strömungsprozesse ………........................................................................ 80

$4.7 \quad$ Fragen und Übungen ............................................................................... 82

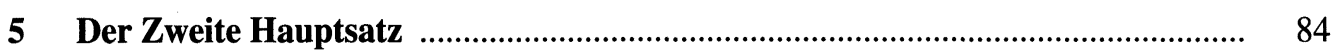

5.1 Aussagen über Prozesse ............................................................................ 84

5.2 Entropie und Entropiestrom .................................................................. 88

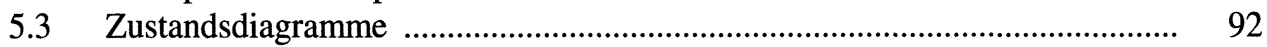

$5.4 \quad$ Energieumwandlung ................................................................................... 99

$5.5 \quad$ Exergie und Anergie ............................................................................. 106

$5.6 \quad$ Fragen und Übungen .............................................................................. 112 
6 Zustandsgleichungen Idealer Gase ....................................................... 117

6.1 Gasgleichung, Gaskonstanten, Normmolvolumen .................................... 117

6.2 Kalorische Zustandsgleichungen .......................................................... 120

6.3 Entropie und Entropiediagramme .......................................................... 124

6.4 Wärmekapazitäten und Isentropenexponent .............................................. 128

6.5 Fragen und Übungen ..................................................................... 132

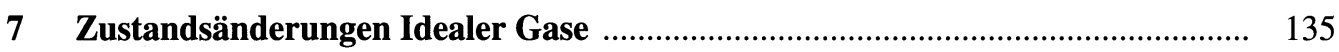

7.1 Allgemeine und spezielle Zustandsänderungen ..................................... 135

Isobare Zustandsänderung .................................................................. 136

7.3 Isochore Zustandsänderung ....................................................................... 139

Isotherme Zustandsänderung .................................................................. 140

7.5 Isentrope Zustandsänderung ................................................................ 143

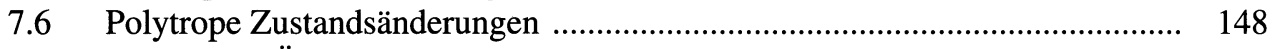

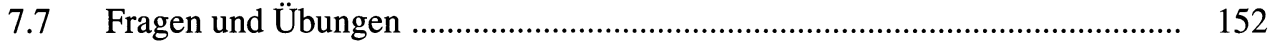

8 Gas- und Gas-Dampf-Gemische ............................................................. 156

8.1 Anteile und Teilgrößen von Gasgemischen, DALTON`sches Gesetz ............ 156

8.2 Gasgleichung, Gaskonstanten und Molmassen von Gasgemischen ................ 158

8.3 Kalorische Zustandsgrößen von Gasgemischen .......................................... 160

8.4 Gas-Dampf-Gemische, Feuchte Luft ........................................................ 162

8.5 Zustandsgrößen und Zustandsdiagramme feuchter Luft ............................. 164

8.6 Luftbehandlungsanlagen ................................................................. 170

8.7 Mischen, Erwärmen und Kühlen feuchter Luft ......................................... 171

8.8 Einsprühen von Wasser in feuchte Luft .................................................... 176

8.9 Verdunstung und Taubildung ................................................................. 179

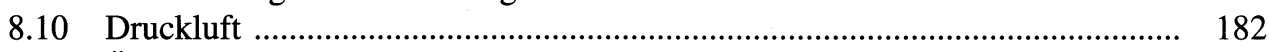

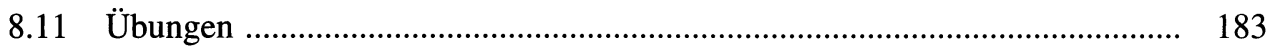

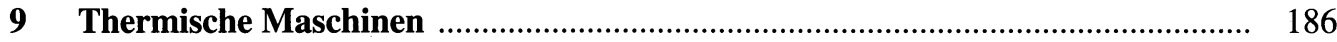

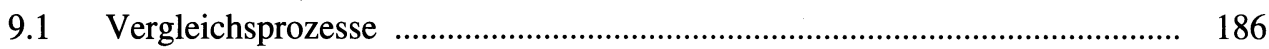

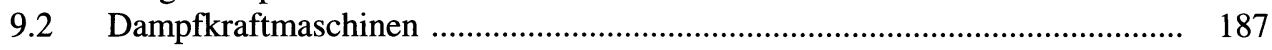

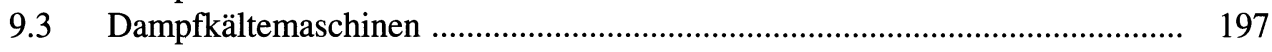

9.4 Verbrennungsmotoren ........................................................................ 201

9.5 Gasturbinen ................................................................................ 204

9.6 Gaskältemaschinen ............................................................................. 209

$9.7 \quad$ Verbundkraftwerke .............................................................................. 212

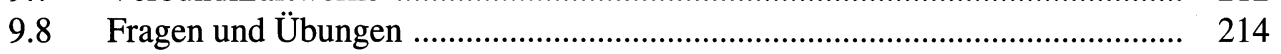

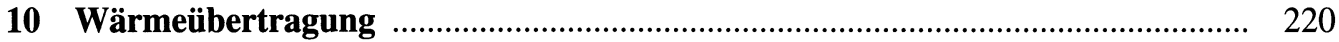

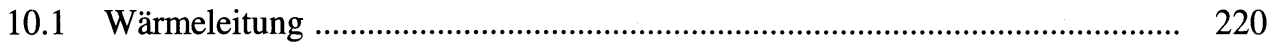

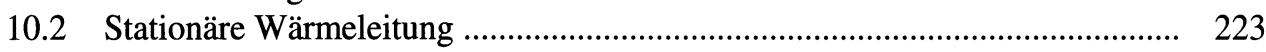

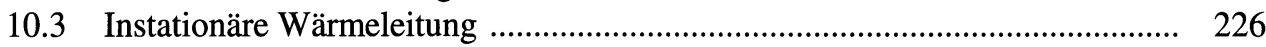

10.4 Numerische Lösungsmethoden ............................................................ 230

10.5 Konvektiver Wärmeübergang .................................................................. 234

10.6 Wärmeübergang bei erzwungener Konvektion .......................................... 238

10.7 Wärmeübergang bei freier Konvektion .................................................... 240

10.8 Wärmeübergang bei Phasenänderung ....................................................... 243

10.9 Wärmestrahlung ............................................................................ 246

10.10 Wärmestrahlung zwischen festen Oberflächen ......................................... 251 


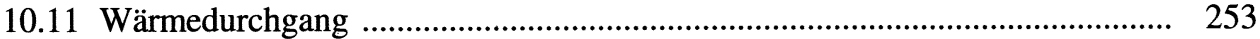

10.12 Wärmeaustausch im Gleichstrom und Gegenstrom ................................. 255

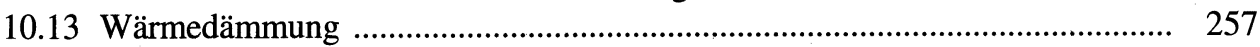

10.14 Fragen und Übungen ..................................................................... 261

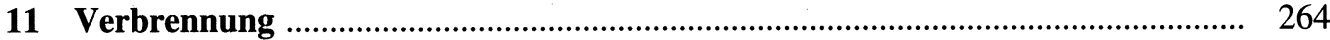

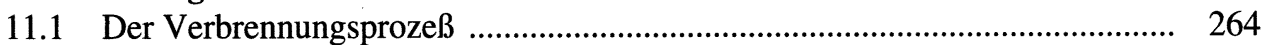

11.2 Brennstoffe, Brennluft und Grundreaktionen ........................................... 265

11.3. Sauerstoffbedarf, Luftbedarf, Verbrennungsgasanfall ............................... 267

11.4 Brennwert und Heizwert .................................................................... 275

11.5 Übungen ....................................................................................... 280

Tabellen (mit Griffstreifen) ........................................................................... 282

T-1 Einheiten und Einheitenumrechnung ................................................... 282

T-1a Universelle Konstanten und Normzustand .............................................. 283

T-2 Angelsächsische Einheiten ................................................................... 283

T-3 Stoffwerte Idealer Gase ......................................................................... 284

T-4 Mittlere molare Wärmekapazitäten ....................................................... 285

T-5 Sättigungsdampftafel für Wasser (Temperaturtafel) ................................ 286

T-6 Sättigungsdampftafel für Wasser (Drucktafel) ......................................... 288

T-6a Zustandsgrößen für ungesättigte Wasserflüssigkeit und überhitzten Wasserdampf ....................................................................................... 290

T-7 Sättigungsdampftafel für Ammoniak ...................................................... 292

T-8a Sättigungsdampftafel für R134a ........................................................... 293

T-8 MoLLIER-Druck-Enthalpie-Diagramm für R134a ....................................... 294

T-9 Stoffwerte gesättigter feuchter Luft ....................................................... 296

T-10 Thermophysikalische Stoffgrößen .......................................................... 297

T-11 Zahlenwerte der GAUSS'schen Fehlerfunktion ............................................ 299

T-12 Emissionsgrade technischer Oberflächen .................................................. 300

T-13 Feste Brennstoffe ............................................................................. 300

T-14 Flüssige Brennstoffe I ....................................................................... 300

T-15 Flüssige Brennstoffe II .......................................................................... 301

T-16 Gasförmige Brennstoffe I ............................................................... 301

T-17 Gasförmige Brennstoffe II ............................................................. 301

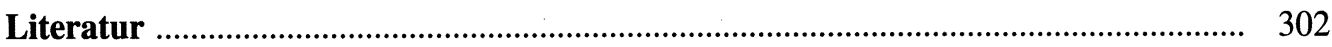

Sachwortverzeichnis (deutsch/englisch) ........................................................... 304

Hinweise zur CD-ROM ........................................................................................ 319

CD-ROM Umschlagtasche

LV-BERECHNUNGSSOFTWARE

INSTALLATION

ENGLISCH-DEUTSCHES SACHWORTVERZEICHNIS

THERMODYNAMIK MEMORY

THERMODYNAMIK GLOSSAR

MOLLIER-Enthalpie-Entropie-Diagramm für Wasserdampf 


\section{Verzeichnis der Beispiele}

Beispiel Seite Stichwort

\begin{tabular}{lll}
\hline 1.1 & 5 & Größengleichung \\
1.2 & 6 & Berechnungsmuster
\end{tabular}

$\begin{array}{lll}2.1 & 13 & \text { Systemabgrenzung } \\ 2.2 & 17 & \text { Volumen } \\ 2.3 & 19 & \text { Massenstrom } \\ 2.4 & 23 & \text { Druck } \\ 2.5 & 24 & \text { Temperatur } \\ 2.6 & 28 & \text { Quasistatische Zustandsänderung } \\ 2.7 & 29 & \text { Nichtstatische Zustandsänderung }\end{array}$

$2.7 \quad 29 \quad$ Nichtstatische Zustandsänderung

$\begin{array}{lll}3.1 & 33 & \text { Thermische Dehnung } \\ 3.2 & 42 & \text { Dampftafel } \\ 3.3 & 42 & \text { Naßdampf } \\ 3.4 & 43 & \text { Verdampfung } \\ 3.5 & 50 & \text { Gasgleichung } \\ 3.6 & 51 & \text { Gasmasse } \\ 3.7 & 51 & \text { Druckänderung } \\ 3.8 & 56 & \text { Stoffgemisch }\end{array}$

$\begin{array}{lll}4.1 & 73 & \text { Wärmeaustauscher } \\ 4.2 & 79 & \text { Dampfkraftwerk } \\ 4.3 & 79 & \text { Dampferzeugung } \\ 4.4 & 81 & \text { Rohrströmung } \\ & & \\ 5.1 & 91 & \text { Fanno-Linien } \\ 5.2 & 94 & \text { Dampfkraftmaschine } \\ 5.2 \mathrm{a} & 96 & \text { Dampfkraftprozeß } \\ 5.2 \mathrm{~b} & 97 & \text { Kältemittelflasche } \\ 5.3 & 105 & \text { Thermoelement } \\ 5.4 & 111 & \text { Exergetischer Wirkungsgrad } \\ 5.5 & 111 & \text { Exergieverlust } \\ 5.6 & 111 & \text { Exergieverlust } \\ & & \\ 6.1 & 119 & \text { Gasvolumen } \\ 6.2 & 119 & \text { Gasdichte } \\ 6.3 & 131 & \text { Wärmekapazität }\end{array}$

\section{Beispiel Seite Stichwort}

\begin{tabular}{lll}
\hline 7.1 & 136 & Luftverdichtung \\
7.2 & 138 & Isobare Zustandsänderung \\
7.3 & 140 & Isochore Zustandsänderung \\
7.4 & 142 & Isotherme Zustandsänderung \\
7.5 & 146 & Druckluftanlage \\
7.6 & 151 & Druckluftmotor \\
7.7 & 152 & Versuchsauswertung \\
& & \\
8.1 & 159 & Gasgemisch \\
8.2 & 161 & Gasgemisch \\
8.3 & 166 & Diagramm für feuchte Luft \\
8.4 & 173 & Luftmischung \\
8.5 & 174 & Lufterwärmung \\
8.6 & 176 & Luftkühlung \\
8.7 & 178 & Randmaßstab \\
8.8 & 178 & Klimaanlage \\
8.9 & 182 & Druckluft \\
& & \\
9.1 & 195 & Industriedampfanlage \\
9.2 & 200 & Gewerbekälteanlage \\
9.3 & 203 & Wirkungsgrade von \\
& & Verbrennungsmotoren \\
9.4 & 206 & Wirkungsgrad einer \\
& & Gasturbinenanlage \\
9.5 & 214 & Wirkungsgrad von \\
& & Verbundkraftwerken \\
& &
\end{tabular}

$10.1 \quad 228 \quad$ Aluminiumkugel

$10.2 \quad 230$ Halbunendlicher Körper

10.3246 Wärmeübergang am Rohr

10.4253 Wärmestrahlung

10.5260 Wärmedurchgang

$11.1 \quad 270 \quad$ Kohleverbrennung

$11.2 \quad 274 \quad$ Erdgasverbrennung

11.3279 Verbrennungstemperatur 


\section{Formelzeichen}

In den in eckigen Klammern angegebenen Abschnitten werden die Größen erstmals erwähnt oder ausführlich behandelt.

\section{Größen für die Thermodynamik}

\section{Lateinische Zeichen}

A Fläche

b Breite

$B \quad$ Anergie [5.5]

$b \quad$ spezifische Anergie [5.5]

$B_{\mathrm{m}} \quad$ molare Anergie [5.5]

$\dot{B} \quad$ Anergiestrom [5.5]

$B_{\mathrm{H}} \quad$ Anergie der Enthalpie [5.5]

$B_{\mathrm{Q}} \quad$ Anergie der Wärme [5.5]

c Geschwindigkeit [2.4]

C Wärmekapazität [6.4]

$c_{\mathrm{p}} \quad$ spez. isobare Wärmekapazität [6.4]

$c_{\mathrm{v}} \quad$ spez. isochore Wärmekapazität [6.4]

$c_{\mathrm{n}} \quad$ spez. polytrope Wärmekapazität [6.4]

$C_{\mathrm{mp}} \quad$ molare isobare Wärmekapazität [6.4]

$C_{\mathrm{mv}} \quad$ molare isochore Wärmekapazität [6.4]

$C_{\rho \mathrm{p}} \quad$ volumetr. isobare Wärmekapazität [6.4]

$C_{\mathrm{pv}}$ volumetr. isochore Wärme-

kapazität [6.4]

$\bar{C} \quad$ mittlere Wärmekapazität [6.4]

$c_{\mathrm{fl}} \quad$ spezif. Wärmekapazität v. Flüssigk. [4.4]

$c_{\mathrm{w}} \quad$ spezif. Wärmekapazität v. Wasser [4.4]

$d, D \quad$ Durchmesser

$E_{\text {kin }} \quad$ kinetische Energie [4.1]

$E_{\text {pot }}$ potentielle Energie [4.1]

$E$ Exergie [5.5]

$e \quad$ spezifische Exergie [5.5]

$E_{\mathrm{m}} \quad$ molare Exergie [5.5]

$\dot{E} \quad$ Exergiestrom [5.5]

$E_{\mathrm{H}} \quad$ Exergie der Enthalpie [5.5]

$E_{\mathrm{Q}} \quad$ Exergie der Wärme [5.5]

$E_{\mathrm{U}} \quad$ Exergie der Inneren Energie [5.5]

$E_{\mathrm{v}} \quad$ Exergieverlust [5.5]

$F \quad$ Kraft [4.2]

$g \quad$ Fallbeschleunigung [4.1]

$H \quad$ Enthalpie [4.4]

$h$ spezifische Enthalpie [4.4]

$H_{\mathrm{m}} \quad$ molare Enthalpie [4.4]

$\dot{H} \quad$ Enthalpiestrom [4.4]

$\Delta h_{\mathrm{d}} \quad$ (spez.) Verdampfungs-/Verflüssigungsenthalpie [4.4]

$\Delta h_{\mathrm{f}} \quad$ (spez.) Schmelz-/Erstarrungsenthalpie [4.4]

$\Delta h_{\text {sub }} \quad$ (spez.) Sublimations-/Desublimationsenthalpie [4.4]

$H_{\mathrm{u}} \quad$ Heizwert [11.4]

$H_{\text {um }}$ molarer Heizwert [11.4]

$H_{\mathrm{uv}} \quad$ volumetrischer Heizwert [11.4]

$H_{\mathrm{o}} \quad$ Brennwert [11.4]

$H_{\text {om }} \quad$ molarer Brennwert [11.4]

$H_{\text {ov }} \quad$ volumetrischer Brennwert [11.4]
I Massenstromdichte,

Massengeschwindigkeit [2.4]

$J \quad$ Streuenergie [4.1]

$j \quad$ spezifische Streuenergie [4.1]

$J_{\mathrm{m}} \quad$ molare Streuenergie [4.1]

$j \quad$ Streuenergiestrom [4.1]

$l, L \quad$ Länge

$\ell \quad$ Luftbedarf

(auf Brennstoffmasse bezogen) [11.3]

$\ell_{\min } \quad$ Mindestluftbedarf

(auf Brennstoffmasse bezogen) [11.3]

$\ell_{\mathrm{m}} \quad$ molarer Luftbedarf

(auf Brennstoffmasse bezogen) [11.3]

$\left(\ell_{\mathrm{m}}\right)_{\min }$ molarer Mindestluftbedarf (auf Brennstoffmasse bezogen) [11.3]

$L \quad$ molarer Luftbedarf (auf BrennstoffStoffmenge bezogen) [11.3]

$L_{\min } \quad$ molarer Mindestluftbedarf (auf Brennstoff-Stoffmenge bezogen) [11.3]

$m \quad$ Masse [2.4]

$\dot{m} \quad$ Massenstrom [2.4]

$M \quad$ Molmasse [2.4]

$M_{\mathrm{g}} \quad$ Gemisch-Molmasse [8.2]

$n \quad$ Stoffmenge [2.4]

$\dot{n} \quad$ Stoffmengenstrom [2.4]

$n \quad$ Polytropenexponent [7.6]

$N_{\mathrm{A}} \quad$ AvOGADRO-Konstante [2.4]

$o_{\min } \quad$ Mindestsauerstoffbedarf (auf Brennstoffmasse bezogen) [11.3]

$\left(o_{\mathrm{m}}\right)_{\min }$ molarer Mindestsauerstoffbedarf (auf Brennstoffmasse bezogen) [11.3]

$O_{\text {min }} \quad$ molarer Mindestsauerstoffbedarf (auf

Brennstoff-Stoffmenge bezogen) [11.3]

$p \quad$ Druck [2.5]

$p_{\text {amb }} \quad$ Umgebungs/Atmosphärendruck [2.5]

$p_{\mathrm{e}} \quad$ Überdruck, effektiver Druck [2.5]

$p_{\mathrm{n}} \quad$ Normdruck [2.5]

$p_{\mathrm{kr}} \quad$ kritischer Druck [3.3]

$p_{\text {tr }} \quad$ Tripelpunktsdruck [3.4]

$p^{\prime} \quad$ Sättigungsdampfdruck [3.4]

$P \quad$ Arbeitsleistung $[1.4,4.1]$

$Q \quad$ Wärme [4.1, 4.3]

$q \quad$ spezifische Wärme $[4.1,4.3]$

$Q_{\mathrm{m}} \quad$ molare Wärme [4.3]

$\dot{Q} \quad$ Wärmestrom $[4.1,4.3]$

$\dot{Q}_{\text {o }} \quad$ beim unteren Temperaturniveau einer Maschine übertragener Wärmestrom, Kälteleistung [5.4]

$\dot{Q}_{c} \quad$ beim oberen Temperaturniveau einer Kältemaschine übertragener

Wärmestrom [5.4]

$\dot{Q}_{\text {WP }} \quad$ beim oberen Temperaturniveau einer Wärmepumpe übertragener

Wärmestrom, Heizleistung [5.4] 


\begin{tabular}{|c|c|}
\hline$q_{\mathrm{f}}$ & Flüssigkeitswärme [9.2] \\
\hline$q_{\mathrm{u}}$ & Überhitzungswärme [9.2] \\
\hline$r_{\mathrm{i}}$ & Raumanteil (der Komponente $\mathrm{i}$ eines \\
\hline & Gemisches) [8.1] \\
\hline & Verdampfungswärme [9.2] \\
\hline$r, R$ & Radius \\
\hline$R$ & (spezifische) Gaskonstante $[3.6,6.1]$ \\
\hline$R_{\mathrm{m}}$ & molare Gaskonstante [6.1] \\
\hline$R_{\mathrm{i}}$ & $\begin{array}{l}\text { (spezifische) Gaskonstante } \\
\text { (der Gemisch-Komponente i) [8.1] }\end{array}$ \\
\hline$R_{\mathrm{g}}$ & (spezifische) Gemisch-Gaskonstante [8.2] \\
\hline & Entropie [5.2] \\
\hline & spezifische Entropie [5.2] \\
\hline$S_{\mathrm{m}}$ & molare Entropie [5.2] \\
\hline$\dot{S}$ & Entropiestrom [5.2] \\
\hline$\dot{S}_{\mathrm{Q}}$ & Entropiestrom durch \\
\hline & Wärmeübertragung [5.2] \\
\hline$\dot{S}_{\mathrm{J}}$ & Entropiestromerzeugung durch \\
\hline & (empirische) Temperatur [2.5] \\
\hline$T$ & (thermodynamische) Temperatur [2.5] \\
\hline$T_{\mathrm{n}}$ & Normtemperatur [2.5] \\
\hline$T_{\mathrm{kr}}, t_{\mathrm{kr}}$ & kritische Temperatur [3.3] \\
\hline$T_{\mathrm{tr}}, t_{\mathrm{tr}}$ & Tripelpunktstemperatur [3.5] \\
\hline$T_{0}, t_{0}$ & $\begin{array}{l}\text { unteres Temperaturniveau } \\
\text { thermischer Maschinen [5.4] }\end{array}$ \\
\hline$T_{\mathrm{c}}, t_{\mathrm{c}}$ & $\begin{array}{l}\text { oberes Temperaturniveau von } \\
\text { Kältemaschinen [5.4] }\end{array}$ \\
\hline$T_{\mathrm{u}}$ & Umgebungstemperatur \\
\hline $\bar{\tau}$ & $\begin{array}{l}\text { mittlerer Wert der Temperatur } \\
\text { der Wärmeübertragung }[6.4,9.2]\end{array}$ \\
\hline$\left\langle t_{\mathrm{V}}\right\rangle_{\mathrm{ad}}$ & adiabate Verbrennungstemp. [11.4] \\
\hline$U$ & Innere Energie [4.3] \\
\hline$u$ & spezifische Innere Energie [4.3] \\
\hline$U_{\mathrm{m}}$ & molare Innere Energie [4.3] \\
\hline$V$ & (extensives) Volumen [2.4] \\
\hline$v$ & spezifisches Volumen [2.4] \\
\hline$V_{\mathrm{m}}$ & molares Volumen, Molvolumen [2.4] \\
\hline$\dot{V}$ & Volumenstrom [2.4] \\
\hline$v_{\mathrm{kr}}$ & spezifisches kritisches Volumen [3.3] \\
\hline$W$ & Ärbeit $[4.1,4.2]$ \\
\hline$w$ & beit $[4.1,4.2]$ \\
\hline$W_{\mathrm{m}}$ & molare Arbeit $[4.1,4.2]$ \\
\hline$W_{\mathrm{v}}$ & Volumenarbeit $[4.1,4.2]$ \\
\hline$W_{\mathrm{vs}}$ & Schubarbeit [4.2] \\
\hline$W_{\mathrm{p}}$ & Druckarbeit [4.2] \\
\hline$W_{\mathrm{t}}$ & Technische Arbeit $[4.1,4,2]$ \\
\hline$W_{\mathrm{H}}$ & Hubarbeit [4.2] \\
\hline$W_{\mathrm{B}}$ & Beschleunigungsarbeit [4.2] \\
\hline$W_{\mathrm{K}}$ & Kreisprozeßarbeit [4.5] \\
\hline$x_{\mathrm{d}}$ & Dampfgehalt (von $\mathrm{NaBdamp}$ \\
\hline$x_{\mathrm{f}}$ & Flüssigkeitsgehalt (von Schmelze) [3.5] \\
\hline$x_{\mathrm{s}}$ & Dampfgehalt (v. Sublimationsstaub) [3.5] \\
\hline & Wassergehalt (feuchter Luft) [8.5] \\
\hline$x^{\prime}$ & $\begin{array}{l}\text { Wassergehalt gesätt. feuchter Luft, } \\
\text { Sättigungswassergehalt [8.5] }\end{array}$ \\
\hline & Ortshöhe, Höhe über Bezugsniveau \\
\hline & Realgasfaktor [3.6] \\
\hline
\end{tabular}

\section{Griechische Zeichen}

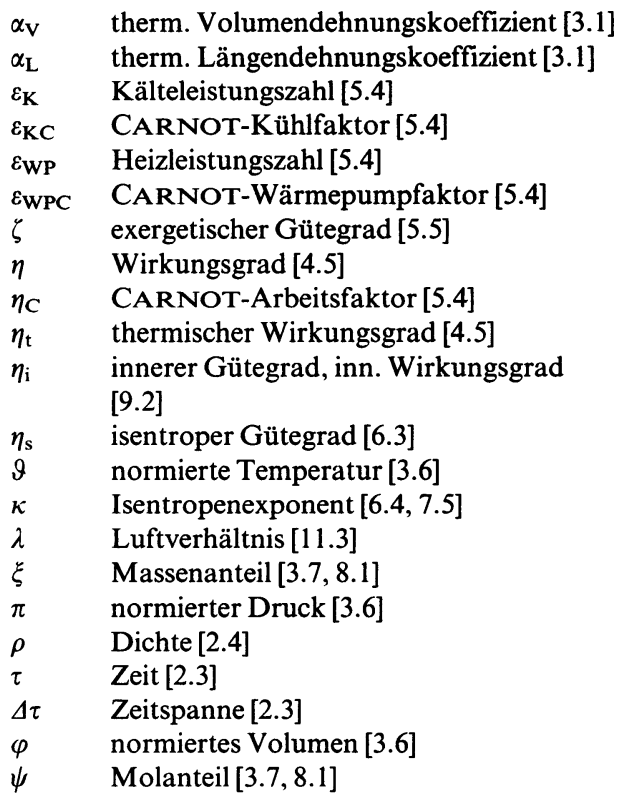

\section{Indizes}

$\begin{array}{ll}\text { 1 }(2,3, \ldots) & \text { im Zustand 1 }(2,3, \ldots)[4.1] \\ 12(23, \ldots) & \text { bei der Zustandsänderung } \\ & 1-2(2-3, \ldots)[4.1] \\ \text { amb } & \text { der Umgebung } \\ \text { B } & \text { für die Beschleunigung [4.2] } \\ \text { B } & \text { von Brennstoff [1.2, 11.2] } \\ \text { c } & \text { beim oberen Temperaturniveau } \\ & \text { einer Kältemaschine [5.4] } \\ \text { C } & \text { bei CARNOT-Bedingungen [5.4] } \\ \text { d } & \text { im Naßdampfgebiet [3.4] } \\ \text { e } & \text { effektiv [2.5] } \\ \text { e } & \text { eines Elementes [3.7] } \\ \text { f } & \text { im Schmelzgebiet [3.5] } \\ \text { g } & \text { eines Gemisches [3.7] } \\ \text { H } & \text { der Enthalpie [5.5] } \\ \text { H } & \text { beim Hub [4.2] } \\ \text { i } & \text { einer Komponente [3.7] } \\ \text { K } & \text { des Kreisprozesses [4.5] } \\ \text { kr } & \text { im kritischen Zustand [3.3] } \\ \text { L } & \text { von Luft [1.3] } \\ \text { m } & \text { molar [2.3] } \\ \text { n } & \text { Normwert [2.6] } \\ \text { o } & \text { Bezugswert, unteres Temperatur- oder } \\ & \text { Druckniveau therm. Maschinen [5.4] } \\ \text { o } & \text { unteres Temperaturniveau } \\ \text { p } & \text { einer Kältemaschine [5.4] } \\ \text { bei konstantem Druck [6.4] } & \text { der Wärme [5.5] } \\ \text { s, sub } & \text { bei Sublimation [3.5, 4.4] } \\ \text { s } & \begin{array}{l}\text { bei konstanter Entropie [6.3] } \\ \text { bei konstanter Temperatur [6.4] }\end{array} \\ & \end{array}$


T der Turbine [6.3, 9.5]

tr in einem Tripelzustand [3.5]

u.U der Umgebung [5.4]

$\mathrm{v}$ bei konstantem Volumen

$v \quad$ des Verdichters [6.3, 9.5]

$\mathrm{V} \quad$ der Verbrennung [11.4]

w von Wasser [4.4]

wQ der Wärmequelle [5.4]

ws der Wärmesenke [5.4]

WKM der Wärmekraftmaschine [5.4]

$\rho \quad$ volumetrisch [6.6]

\section{Hochzeichen}

$\begin{array}{ll}- & \text { Stromgröße [2.3] } \\ - & \text { mittlerer Wert [6.4, 9.2] } \\ & \text { gesättigte Flüssigkeit [3.4] } \\ \prime & \begin{array}{l}\text { Sattdampf, trocken gesättigter/ } \\ \text { kondensierender Dampf [3.4], } \\ \text { desublimierender Dampf [3.5] }\end{array} \\ * & \begin{array}{l}\text { schmelzender Feststoff/Eis [3.5], } \\ \text { sublimierender Feststoff/Eis [3.5] } \\ \text { erstarrende Flüssigkeit [3.5] }\end{array}\end{array}$

\section{Zusätzliche Größen für die Wärmeübertragung}

\section{Lateinische Zeichen}

$a \quad$ Temperaturleitfähigkeit [10.1]

a Absorptionsgrad [10.9]

$a_{\lambda} \quad$ spektraler Absorptionsgrad [10.9]

$b \quad$ Wärmeeindringkoeffizient [10.3]

Bi BIOT-Zahl [10.3]

c Lichtgeschwindigkeit [10.9]

$C_{12} \quad$ Strahlungsaustauschzahl [10.10]

$d \quad$ Durchlaßgrad [10.9]

$d_{\mathrm{h}} \quad$ hydraulischer Durchmesser [10.6]

$\dot{E} \quad$ in den Halbraum emittierter Wärmestrom pro Fläche [10.9]

$\dot{E}_{\mathrm{n}} \quad$ emittierter Wärmestrom in Richtung der

Flächennormalen pro Fläche

und Raumwinkeleinheit [10.9]

$\dot{E}_{\beta} \quad$ emittierter Wärmestrom im Winkel $\beta$ zur

Flächennormalen pro Fläche

und Raumwinkeleinheit [10.9]

$f \quad$ verschiedene numerische Verfahren

beschreibender Faktor [10.4]

Fo FOURIER-Zahl [10.3]

$\mathrm{Ga}$ GALILEI-Zahl [10.8]

Gr GRASHOF-Zahl [10.5]

$h \quad$ PLANCKsches Wirkungsquantum [10,9]

I spektrale Strahlungsintensität [10.9]

$I_{\text {el }} \quad$ elektrischer Strom [10.2]

$k$ BOLTZMANN-Konstante [10.9]

$k \quad$ Wärmedurchgangskoeffizient [10.11]

$L \quad$ charakteristische Abmessung [10.3]

$m \quad$ Maßstabsfaktor [10.5]

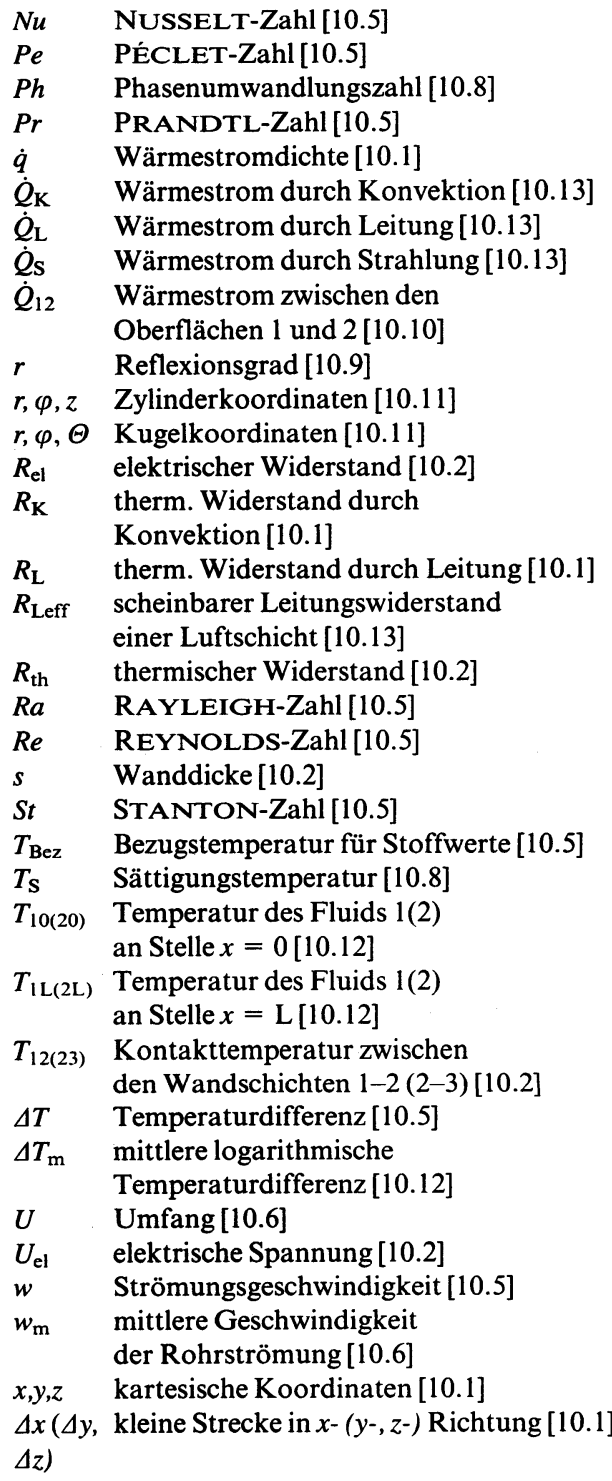

\section{Griechische Zeichen}

$\alpha \quad$ Wärmeübergangskoeffizient [10.1, 10.5]

$\alpha_{\text {Str }}$ Strahlungsanteil am Wärmeübergangskoeffizienten [10.10]

$\beta \quad$ therm.Ausdehnungskoeffizient $[10.5,10.7]$

$\delta \quad$ Strömungsgrenzschicht-Dicke [10.5]

$\delta_{\mathrm{T}} \quad$ Temperaturgrenzschicht-Dicke [10.5]

$\varepsilon \quad$ Emissionsgrad [10.9]

$\varepsilon_{\mathrm{n}} \quad$ Emissionsgrad in Normalenrichtung [10.9]

$\varepsilon_{\lambda} \quad$ spektraler Emissionsgrad [10.9]

$\eta \quad$ dynamische Viskosität [10.5]

$\Theta, r, \varphi$ Kugelkoordinaten [10.1] 
$\lambda \quad$ Wärmeleitfähigkeit [10.1]

$\lambda \quad$ Wellenlänge [10.9]

$\lambda_{\text {eff }}$ effektive Wärmeleitfähigkeit einer Luftschicht [10.13]

$\lambda_{\text {opt }} \quad$ Wellenlänge der maximalen spektralen Strahlungsintensität [10.9]

$\mu \quad$ dimensionslose Variable [10.3]

$v \quad$ kinematische Viskosität [10.5]

$\sigma_{\mathrm{S}}$ STEFAN-BOLTZMANN-

$$
\text { Konstante [10.9] }
$$

$\tau_{\mathrm{S}} \quad$ Schubspannung [10.5]

$\varphi_{12} \quad$ Einstrahlzahl [10.10]

$\varphi, z, r \quad$ Zylinderkoordinaten [10.1]

$\varphi, \Theta, r$ Kugelkoordinaten [10.1]

\section{Indizes}

a auf der Außenseite [10.2]

A eines Gitterknotenpunktes außerhalb des Körpers [10.4]

A im Rohraustritt [10.6]

D der Dämmschicht [10.13]

E des östl. Nachbarpunktes von $P$ [10.4]

E im Rohreintritt [10.6]

F für Flüssigkeiten [10.6]

i auf der Innenseite [10.2]

i einer Wandschicht [10.2]
I eines ersten inneren Gitterknotenpunktes [10.4]

k für den Umschlag von laminarer in turbulente Strömung [10.6]

1 bei laminarer Strömung [10.6]

$\mathrm{N}$ des nördl. Nachbarpunktes von $\mathrm{P}$ [10.4]

$\mathrm{P} \quad$ eines Gitterknotenpunktes [10.4]

R der Rohrwand [10.13]

S des schwarzen Körpers [10.9]

S des südl. Nachbarpunktes von P [10.4]

$\mathrm{t}$ bei turbulenter Strömung [10.6]

w an der Wand [10.1]

W des westl. Nachbarpunktes von $P$ [10.4]

$\mathrm{x}(\mathrm{y}, \mathrm{z}) \quad \mathrm{x}-(\mathrm{y}-, \mathrm{z}-)$ Komponente [10.1]

$\mathrm{x}(\mathrm{y}, \mathrm{z})$ am $\operatorname{Ort} \mathrm{x}(\mathrm{y}, \mathrm{z})[10.1]$

$0 \quad$ zum Zeitpunkt Null [10.3]

1 (2) der Oberfläche 1 (2) [10.10]

l(2) des Fluids 1 (2) [10.11]

$\infty \quad$ im Fluid weitab der Grenzschicht $[10.1,10.5]$

\section{Hochzeichen}

n am Ende eines Zeitschrittes [10.4]

o $\quad$ zu Beginn eines Zeitschrittes [10.4]

$\tau \quad$ während eines Zeitschrittes [10.4] 